\title{
Time Resolved Photoluminescence of Si-doped High Al Mole Fraction AlGaN Epilayers Grown by Plasma-Enhanced Molecular Beam Epitaxy
}

\author{
Madalina Furis, Alexander N. Cartwright, Jeonghyun Hwang ${ }^{1}$ and William J. Schaff ${ }^{1}$, \\ Department of Electrical Engineering, University at Buffalo, \\ Buffalo, NY, 14260, USA \\ ${ }^{1}$ Department of Electrical Engineering, Cornell University, \\ Ithaca, NY, 14853 USA
}

\begin{abstract}
We report on detailed temperature dependent, time-resolved photoluminescence (TRPL) studies of Si-doped AlGaN epilayers. In these samples, the Al concentration varies from $25 \%$ to $66 \%$. The samples were found to exhibit metallic-like temperature-independent conductivity. The deep level "yellow" emission, whose presence would indicate the existence of a large number of defects associated with growth, Si incorporation, and/or alloy formation, is absent. In addition to emission corresponding to the donor-bound exciton, the PL spectrum exhibits features associated with transitions involving localized carriers. This assignment of the emission mechanisms is based on the activation energies extracted from the temperature dependent photoluminescence (PL) quenching. Specifically, at room temperature the PL spectrum is dominated by transitions involving localized states. The localization energy varied from sample to sample and was observed to be between $115 \mathrm{meV}$ to $200 \mathrm{meV}$. The PL intensity decay in the lower Al mole fraction epilayers exhibits a slow component associated with the presence of donor-bound excitons.
\end{abstract}

\section{INTRODUCTION}

Despite the significant progress made in the development of UV emitters, the fabrication of UV LEDs and laser diodes remains a challenging problem due, in part, to the difficulties encountered in the growth of high $\mathrm{Al}$ content AlGaN. Ultimately, AlGaN materials must be grown with reduced defect densities and high levels of both n- and p-type doping. Achieving very high electron concentration in high $\mathrm{Al}$ content $\mathrm{AlGaN}$ is difficult due to formation of compensating deep acceptor states generated by the $\mathrm{Ga}$ or $\mathrm{Al}$ vacancies $\left(\mathrm{V}_{\mathrm{Ga}}\right)[1,2]$ and oxygen DX centers [3]. As a result, $\mathrm{AlGaN}$ doped with $\mathrm{Si}$ reaches a conductive state only for $\mathrm{Si}$ concentration exceeding $10^{18} \mathrm{~cm}^{-3}$. [4]

A significant number of studies have been dedicated to increasing the n-type doping levels in AlGaN[4-6] as well as studying the nature of the compensating acceptors[7,8], DX centers[1,3,9], and Si donors[6,7,10] in these alloys through transport and/or PL studies. Optical studies are especially important for highly doped $\mathrm{AlGaN}$ epilayers that exhibit a metallic behavior even at room temperature[6,7], such as the ones studied by Hwang et al. [6] who reported temperature independent electron concentrations as high as $1.25 * 10^{20} \mathrm{~cm}^{-3}$ in $\mathrm{Al}_{0.5} \mathrm{Ga}_{0.5} \mathrm{~N}$ and $8.5^{*} 10^{19} \mathrm{~cm}^{-3}$ in $\mathrm{Al}_{0.8} \mathrm{Ga}_{0.2} \mathrm{~N}$ epilayers grown by rf plasma-enhanced molecular beam epitaxy.

The present work is an attempt to identify the origins of donor and acceptor states in samples studied by Hwang et al. through temperature dependent photoluminescence studies. A detailed fitting procedure allows the separation of the different components of the PL spectra and 
the energies associated with the PL intensity quenching are identified with various donors and or acceptor binding energies. The donor bound exciton recombination is found to dominate the spectrum of the low Al mole fraction epilayers at low temperatures. The presence of a very shallow Si donor (binding energy equal to a few $\mathrm{meV}$ ) is identified. Time-resolved photoluminescence measurements are used to estimate the carrier lifetime dependence on temperature in these epilayers.

\section{EXPERIMENTAL DETAILS}

The Si-doped AlGaN epilayers were grown in a turbomolecular Varian Gen II MBE system which uses standard effusion cells for the group III elements. An EPI RF plasma source was used for the nitrogen source and 2 in. sapphire wafers with c-plane orientation were used as substrates. After loading into the growth chamber, each wafer was nitrided by nitrogen plasma at $200{ }^{\circ} \mathrm{C}$ for $30 \mathrm{~min}$. and then heated at $830^{\circ} \mathrm{C}$ for AlN nucleation layer growth. The Si-doped AlGaN wafers with different $\mathrm{Al}$ mole fractions and Si fluxes were grown at $800{ }^{\circ} \mathrm{C}$ after the deposition of a 200 $\AA$ AlN buffer layer. The growth rate was almost the same for all wafers, $4000 \AA / \mathrm{h}$. The Al mole fraction was subsequently determined by x-ray measurements. The thickness of all epilayers is $4000 \AA ̊$ and they are completely relaxed. A complete series of epilayers with Al mole fractions varying between $\mathrm{x}=0.25$ and $\mathrm{x}=1$ were grown through this method. Since the shortest excitation wavelength available for the studies presented here was $266 \mathrm{~nm}$, our photoluminescence studies were limited to Al mole fractions smaller than $65 \%$. All the samples, with the exception of the $52 \% \mathrm{Al}$ mole fraction epilayer, exhibit metallic conductivity up to room temperature with measured Hall carrier concentrations in excess of $10^{18} \mathrm{~cm}^{-3}$ and as high as $1.25^{*} 10^{20} \mathrm{~cm}^{-3}$. More details about the growth process and transport measurements results can be found in reference [6]. Preliminary SIMS measurements show that, in addition to silicon, oxygen is present in the sample in concentrations as high as $3.4 * 10^{19} \mathrm{~cm}^{-3}$.

Time-resolved photoluminescence (TRPL) spectra were obtained using conventional time-correlated techniques. The $800 \mathrm{~nm}, 200 \mathrm{fs}$ pulses from a Coherent $250 \mathrm{KHz}$ repetition rate regenerative amplifier (REGA) were frequency tripled to provide a $250 \mathrm{fs}, 266 \mathrm{~nm}$ pulsed excitation beam with an average power of $5 \mathrm{~mW}$. The excitation beam was focused onto the sample to a diameter of approximately $60 \mu \mathrm{m}$. The luminescence signal was dispersed by a Chromex 250IS monochromator and detected by a Hamamatsu C4334 streak camera with a temporal jitter of $50 \mathrm{ps}$. The carrier lifetimes were estimated using an exponential fit to the observed decay data. For the temperature dependent photoluminescence studies, the samples were placed inside a closed cycle refrigerator. The temperature studies were carried out from 15 $\mathrm{K}$ to $300 \mathrm{~K}$.

\section{RESULTS AND DISCUSSION}

A summary of the PL spectra from the samples under study is presented in Figure 1(a). The spectra taken at low temperatures (continuous lines) exhibit more than one feature at energies lower than the epilayer band gap (marked by arrows in the same figure). The red shift of the highest energy feature in the spectra with respect to the bandgap varies from approximately $30 \mathrm{meV}$ in the low Al mole samples to hundreds of meV in the higher Al concentration epilayers. The spectrum of the $65 \%$ sample could not be recorded in its entirety since the highest excitation energy available for this experiment is lower than the nominal bandgap of $\mathrm{Al}_{0.65} \mathrm{Ga}_{0.35} \mathrm{~N}$. The 

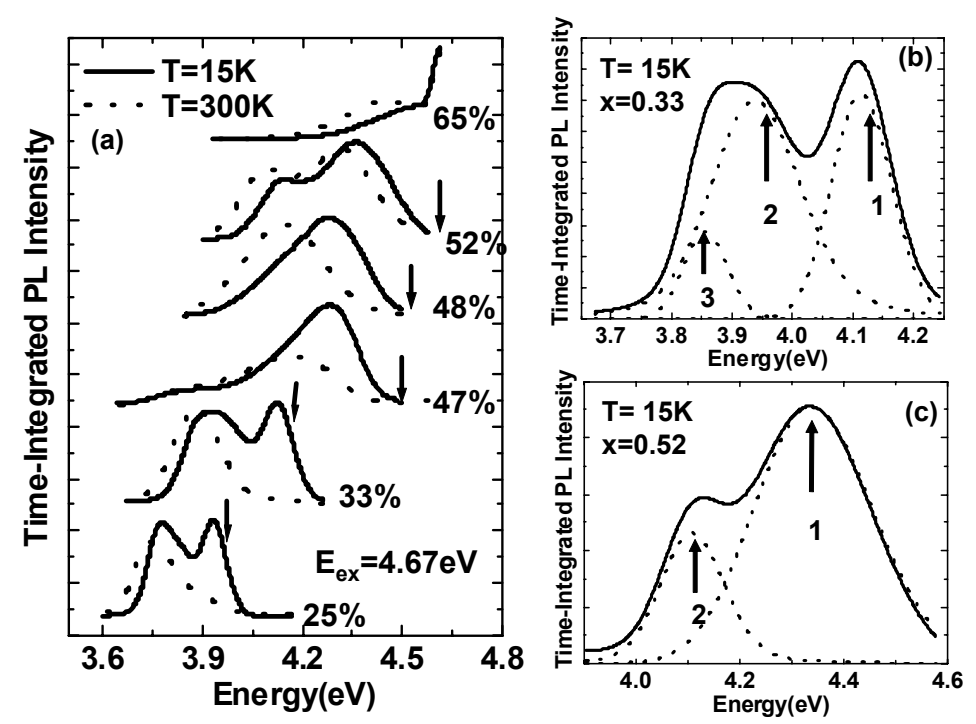

Figure 1 (a) Pl spectra from the samples under study taken at $15 \mathrm{~K}$ (solid lines) and room temperature (dashed lines), (b) Curve fitting of the 15K PL spectrum of sample AL33 using Voigt functions and (c) same fitting for sample AL52.

shape of the spectra changes significantly as the temperature increases such that, in some cases, the feature that dominated the spectrum at low temperature is absent at room temperature (dotted lines). None of the samples exhibit any deep level (yellow) luminescence.

The change in the relative intensity as a function of temperature between the various components in the PL spectra implies the features present in the spectra originate in different recombination mechanisms. This observation suggests a reliable interpretation of the PL spectra from the AlGaN epilayers can be afforded only if each of the components is isolated through a fitting procedure and its temperature dependence is analyzed.

The results of a fitting carried out using Voigt functions for the PL spectra of samples containing 33\% and 52\% Al are plotted in Figure 1(b) and (c). The continuous lines represent the measured spectrum and the dotted curves are the components fitted to the spectrum. In some cases (for example the $33 \%$ or the $25 \%$ Al epilayers) a good fitting was obtained using three functions whereas in others (e.g. the $52 \% \mathrm{Al}$ sample) two components were enough to obtain a good fit to the spectrum.

Next, the integrated intensity of each component was plotted as a function of inverse temperature in an Arhhenius plot and the activation energies associated with the quenching of each component were extracted from the plot, by fitting the intensity dependence on temperature with:

$$
I(T)=\frac{I_{0}}{1+\sum_{i} C_{i} \exp \left(-\frac{E A_{i}}{k_{B} T}\right)}
$$

where $I_{0}$ the represents the time-integrated intensity at low temperatures, and $i=1,2$ or 3 , depending on how many activation energies are associated with each component. The Arhhenius plots for the 25\% sample are shown in Figure 2. 


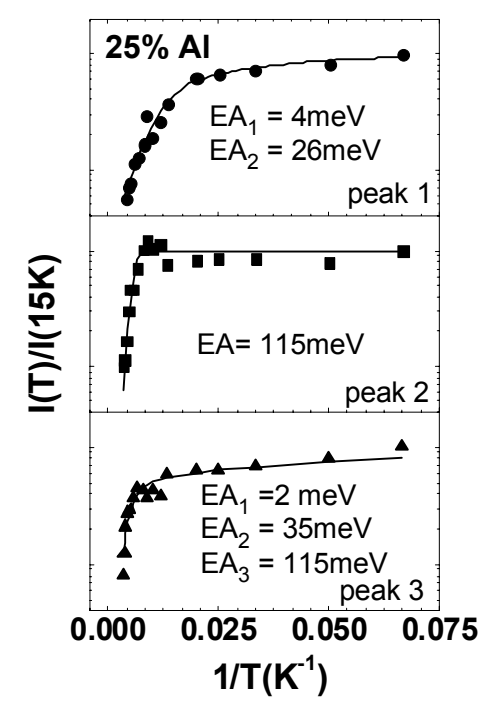

Figure 2 Photoluminescence intensity dependence on temperature for the three transitions identified in the PL spectrum of $\mathrm{Al}_{0.25} \mathrm{Ga}_{0.75} \mathrm{~N}$. The activation energies associated with peak 1 indicate this transition is associated with the radiative recombination of donor bound excitons. The presence of a very shallow donor is also identified.

For the highest energy peak (peak 1) the thermal quenching is characterized by two activation energies equal to $5 \mathrm{meV}$ and $26 \mathrm{meV}$. These energies match the donor bound exciton and free exciton binding energies respectively, suggesting the highest energy feature in the spectrum corresponds to the radiative recombination of donor bound excitons at low temperatures and free excitons at higher temperatures. A similar behavior is encountered in the $33 \% \mathrm{Al}$ sample where peak 1 is also characterized by a low activation energy, of the same order of the PL redshift with respect to the bandgap. In contrast, the second and third peak in the low Al mole fraction samples as well as the PL spectra in the high Al mole fraction samples are characterized by at least one activation energy larger than $100 \mathrm{meV}$. (around $115 \mathrm{meV}$ for the $15 \%$ and $33 \% \mathrm{Al}$ samples and $200 \mathrm{meV}$ for the $47 \%, 48 \%$ and $52 \% \mathrm{Al}$ samples).

The most straight forward approach is to identify the large activation energies with binding energies of shallow acceptors. Such acceptors have been observed in GaN[11], but their origins remain obscure. We cannot exclude the possibility that $\mathrm{Si}$ is substituting for $\mathrm{N}$ as well as $\mathrm{Ga}$, thus acting like an acceptor or the formation of Si clusters with an acceptor character[12]. For the samples exhibiting metallic conductivity the presence of acceptors associated with the formation of $\mathrm{V}_{\mathrm{Ga}} \mathrm{Si}_{\mathrm{Ga}}$ complexes is also possible. [8]

In addition to donor bound excitons and conduction band to acceptor-related features, the photoluminescence spectra contain contributions from donor to acceptors transitions. (for example peak 3 in the $25 \% \mathrm{Al}$ epilayer). The activation energies associated with the donor levels involved in these transitions range between $2 \mathrm{meV}$ and $36 \mathrm{meV}$. Such low numbers indicate the possible formation of band-tail donor like states, almost resonant to the conduction band, and explains the metallic character observed in these samples.

Measurements of the PL intensity decay at different energies across the spectrum confirm the different nature of the recombination mechanisms. Since the epilayers are very thick and completely relaxed, only the recombination of carriers located at the AlN/AlGaN interface and possibly the surface recombination [13] are affected by the presence of spontaneous and piezoelectric polarization. Since the present experiment probes the recombination processes inside the epilayer, such effects can be neglected in the context of the present discussion. The 
photoluminescence intensity decay as a function of time in the $25 \% \mathrm{Al}$ sample is plotted in Figure 3 at three different temperatures and for two different energies. All curves can be fitted by a single exponential with the exception of the decay corresponding to the conduction band to acceptor transition $(3.789 \mathrm{eV})$ decay at low temperatures, which is characterized by two distinct lifetimes. The most interesting aspect of the PL decay at this particular energy is, that the high injection lifetime (measured at early decay times to be $1.1 \mathrm{~ns}$ in this sample) is longer than the low injection (measured at longer decay times) lifetime and not vice versa, as expected. This behavior is encountered only for the donor to acceptor pair recombination in samples that also exhibit excitonic recombination. In contrast all, donor to acceptor transitions in the higher $\mathrm{Al}$ mole fraction samples are characterized by a single exponential decay.

A qualitative explanation for this behavior can be given if we consider that, in a very rough approximation, at low temperatures all non-radiative recombination channels are frozen and the donor-bound excitons recombine leaving the donor in its neutral state. In such a case, at early delay times, the loss of neutral donors through the donor to acceptor pair recombination is compensated by the generation of neutral donors resulting from the exciton recombination. This competition slows the donor-to-acceptor recombination process. Once most of the donor-bound excitons have recombined, the donor to acceptor pair recombination happens on a faster scale.

Temperature studies support this explanation. Figure 3(b) shows that at temperatures above $150 \mathrm{~K}$, where most of the donor-bound excitons no longer exist, (their binding energy equals $5 \mathrm{meV}$ ) the slow component disappears as well. Moreover, this slow component has only been observed in the $25 \%$ and $33 \%$ epilayers, which are the only samples exhibiting a donorbound exciton related feature in the PL spectrum at low temperatures.

\section{CONCLUSIONS}

Component-resolved TRPL studies of Si doped AlGaN epilayers with different Al mole fractions have revealed the presence of a very shallow donor state (binding energy equal to only a few $\mathrm{meV}$ ). The Pl spectra contain several features associated with recombination of donor-bound exciton, conduction band to acceptor and donor-to-acceptor pair recombination. The origins of the acceptor states are still unclear. The slow photoluminescence intensity decay measured at
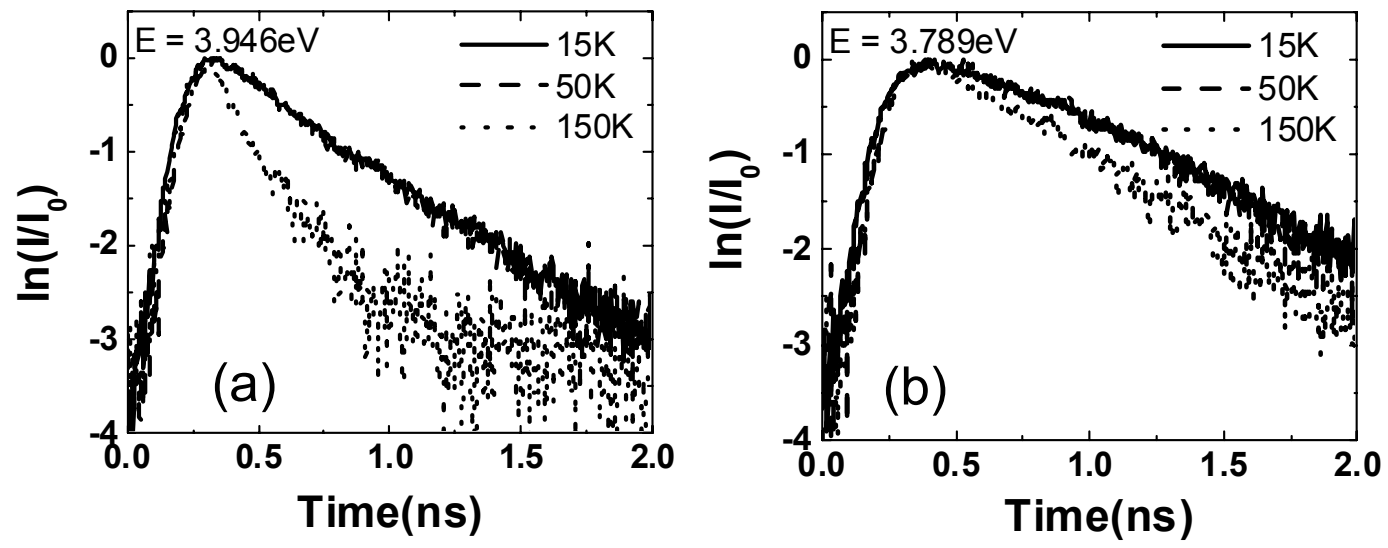

Figure 3 Photoluminescence intensity decay at $15 \mathrm{~K}, 50 \mathrm{~K}$, and $150 \mathrm{~K}$ for the $25 \% \mathrm{Al}$ sample measured at energies corresponding to the excitonic transition (plot(a)) and donor to acceptor transition $(\operatorname{plot}(\mathrm{b}))$. The latter exhibits a slow decay component at low temperatures. The same decay becomes mono-exponential at $150 \mathrm{~K}$. 
early decay times and energies corresponding to the donor-to-acceptor transition in the low $\mathrm{Al}$ mole fraction epilayers is associated with the existence of donor-bound excitons at low temperatures in these samples.

\section{ACKNOWLEDGEMENTS}

This work was supported in part by ANC's NSF CAREER Grant \#9733720, ONR YIP Grant \#N00014-00-1-0508, a Defense University Research Initiative on Nanotechnology Grant \#F496200110358 through the Air Force Office of Scientific Research, and WJS's NSF Grant \#ECS-0123453 and DARPA through Army Research Office Grant \#DAAD19-02-0199.

\section{REFERENCES}

1. C. Stampfl and C. G. Van de Walle, Applied Physics Letters 72 (4), 459 (1998).

2. T. Mattila and R. M. Nieminen, Physical Review B 55 (15), 9571 (1997).

3. M. D. McCluskey, N. M. Johnson, C. G. Van de Walle, D. P. Bour, M. Kneissl, and W. Walukiewicz, Physical Review Letters 80 (18), 4008 (1998).

4. K. B. Nam, J. Li, M. L. Nakarmi, J. Y. Lin, and H. X. Jiang, Applied Physics Letters 81 (6), 1038 (2002).

5. H. Amano and I. Akasaki, Optical Materials 19 (1), 219 (2002); D. Korakakis, H. M. Ng, M. Misra, W. Grieshaber, and T. D. Moustakas, Mrs Internet Journal of Nitride Semiconductor Research 1 (1-46), art. no. (1996); M. Pophristic, S. P. Guo, and B. Peres, Applied Physics Letters 82 (24), 4289 (2003).

6. J. H. Hwang, W. J. Schaff, L. F. Eastman, S. T. Bradley, L. J. Brillson, D. C. Look, J. Wu, W. Walukiewicz, M. Furis, and A. N. Cartwright, Applied Physics Letters 81 (27), 5192 (2002).

7. A. Y. Polyakov, M. Shin, J. A. Freitas, M. Skowronski, D. W. Greve, and R. G. Wilson, Journal of Applied Physics 80 (11), 6349 (1996).

8. M. A. Reshchikov, H. Morkoc, S. S. Park, and K. Y. Lee, Applied Physics Letters 81 (26), 4970 (2002).

9. R. Zeisel, M. W. Bayerl, S. T. B. Goennenwein, R. Dimitrov, O. Ambacher, M. S. Brandt, and M. Stutzmann, Physical Review B 61 (24), R16283 (2000).

10. A. Y. Polyakov, N. B. Smirnov, A. V. Govorkov, M. G. Mil'vidskii, J. M. Redwing, M.Shin, M. Skowronski, D. W. Greve, and R. G. Wilson, Solid-State Electronics 42 (4), 627 (1998).

11. M. A. Reshchikov and R. Y. Korotkov, Physical Review B 64 (11), art. no.115205 (2001);

D. C. Reynolds, D. C. Look, B. Jogai, and R. J. Molnar, Journal of Applied Physics 89

(11), 6272 (2001); G. B. Ren, D. J. Dewsnip, D. E. Lacklison, J. W. Orton, T. S. Cheng, and C. T. Foxon, Materials Science and Engineering B-Solid State Materials for Advanced Technology 43 (1-3), 242 (1997).

12. T. Sato and T. Ishiwatari, Journal of Applied Physics 91 (8), 5158 (2002).

13. O. Ambacher, J. Majewski, C. Miskys, A. Link, M. Hermann, M. Eickhoff, M. Stutzmann, F. Bernardini, V. Fiorentini, V. Tilak, B. Schaff, and L. F. Eastman, Journal of PhysicsCondensed Matter 14 (13), 3399 (2002). 